Article

\title{
High-Density EEG Signal Processing Based on Active-Source Reconstruction for Brain Network Analysis in Alzheimer's Disease
}

\author{
Fabio La Foresta ${ }^{1, *(\mathbb{D})}$, Francesco Carlo Morabito ${ }^{1}\left(\mathbb{D}\right.$, Silvia Marino ${ }^{2}$ and Serena Dattola ${ }^{1}$ (D) \\ 1 DICEAM Department, Mediterranea University of Reggio Calabria, Via Graziella Feo di Vito, \\ 89060 Reggio Calabria, Italy; morabito@unirc.it (F.C.M.); serena.dattola@unirc.it (S.D.) \\ 2 IRCCS Centro Neurolesi Bonino-Pulejo, Via Palermo c/da Casazza, SS. 113, 98124 Messina, Italy; \\ silvia.marino@irccsme.it \\ * Correspondence: fabio.laforesta@unirc.it
}

Received: 30 July 2019; Accepted: 10 September 2019; Published: 13 September 2019 updates

\begin{abstract}
Alzheimer's Disease (AD) is a neurological disorder characterized by a progressive deterioration of brain functions that affects, above all, older adults. It can be difficult to make an early diagnosis because its first symptoms are often associated with normal aging. Electroencephalography (EEG) can be used for evaluating the loss of brain functional connectivity in AD patients. The purpose of this paper is to study the brain network parameters through the estimation of Lagged Linear Connectivity (LLC), computed by eLORETA software, applied to High-Density EEG (HD-EEG) for 84 regions of interest (ROIs). The analysis involved three groups of subjects: 10 controls (CNT), 21 Mild Cognitive Impairment patients (MCI) and 9 AD patients. In particular, the purpose is to compare the results obtained using a 256-channel EEG, the corresponding 10-10 system 64-channel EEG and the corresponding 10-20 system 18-channel EEG, both of which are extracted from the 256-electrode configuration. The computation of the Characteristic Path Length, the Clustering Coefficient, and the Connection Density from HD-EEG configuration reveals a weakening of smallworld properties of MCI and AD patients in comparison to healthy subjects. On the contrary, the variation of the network parameters was not detected correctly when we employed the standard 10-20 configuration. Only the results from HD-EEG are consistent with the expected behavior of the AD brain network.
\end{abstract}

Keywords: complex network analysis; High-Density EEG; multidimensional signal processing; eLORETA

\section{Introduction}

Alzheimer's Disease (AD) is a progressive brain disorder that is the most common cause of dementia among older adults. The first stage of the disease is characterized by a series of symptoms, above all memory problems, which however do not affect daily life activities significantly: this condition is called Mild Cognitive Impairment (MCI). As the disease advances, people experience problems with language, disorientation, and behavioral issues. Gradually, bodily functions are lost, eventually leading to death. An early diagnosis of AD is very difficult because first symptoms are often considered to be consequences of normal aging.

Electroencephalography (EEG) has been used for years as a tool for studying AD [1-3]. It is a non-invasive diagnostic technique, easy-to-use, and relatively low cost. EEG records brain electric activity by means of electrodes placed on the scalp. The potentials measured at the head surface derive from synchronous activity of populations of cortical pyramidal neurons. Signals detected by each electrode result not only from the underlying neurons, but from all active sources, superposed 
as a function of their distance and orientation: this is caused by the conductivity of the scalp and the underlying tissues. Therefore, a realistic head model is fundamental for a correct analysis of EEG signal [4]. EEG has very high temporal resolution, on the order of milliseconds, but a limited spatial resolution. Over the years, many researchers have tried to determine the minimum number of electrodes to avoid undersampling the scalp potential. Several studies have been carried out to set the minimum interelectrode spacing: they obtained a range varying from $1 \mathrm{~cm}$ to $3 \mathrm{~cm}$ [5-7]. For years EEG has been detected by a few electrodes according to the International 10-20 system, with an interelectrode distance of $7 \mathrm{~cm}$. Presently scalp potentials can be recorded using EEG headset which contain up to 256 electrodes (High-Density EEG). Several pieces of research have shown that the limited spatial resolution of standard EEG improves when the number of recording sensors increases [8-12].

Graph theory represents a very useful tool to study complex brain network. According to graph theory, nodes (vertices) denotes the brain regions that are linked by edges, representing structural or functional connection [13]. Brain functional connectivity refers to the functionally integrated relationship between spatially separated brain regions and is defined in terms of statistical dependencies in the time domain (correlation) and in the frequency domain (coherence) among neurophysiological measurements. Coito et al. [14] investigated connectivity in temporal lobe epilepsy (TLE) in the absence of interictal epileptiform discharges (IEDs). Their study revealed a reduced connectivity from regions concordant with the default-mode network and a different network pattern in TLE patients compared to healthy controls. Astolfi et al. [15] employed graph theory for studying HD-EEG and fMRI of healthy subjects during the execution of the Stroop task. Recently, it has been shown that brain networks are characterized by small-world architecture $[13,16]$. The small-world property of a normal brain network combines high levels of local specialization and global integration among nodes and can be measured, respectively, by the Clustering Coefficient (CC) [13] and the Characteristic Path Length $(\lambda)$ [13]. The Connection Density $(D)$ [17] is another index used to define the network's behavior: the more connections there are, the denser the network will be. Several studies reported that the EEG synchrony and the functional connections between regions decrease for AD [18-20]; it has also been proved that small-world properties extracted from EEG of AD patients are very weakened [21-23]. Mammone et al. [24] used the Permutation Disalignment Index (PDI) to obtain the connectivity matrices starting from HD-EEG of three groups: controls subjects, Mild Cognitive Impairment patients, and Alzheimer's disease patients. HD-EEG were also compressed and reconstructed applying the Block Sparse Bayesian Learning (BSBL). It was shown that the three groups have network parameters significantly different and the compression does not alter the results of the network analysis.

The first step for an accurate connectivity analysis is the localization of the brain active sources. In the last two decades, LORETA (Low Resolution Electromagnetic Tomography) has been one of the most popular and widely employed technique for source reconstruction. In particular, LORETA is a linear method for solving the EEG inverse problem which provides a three-dimensional reconstruction of the brain electrical activity. The brain volume is discretized into a volumetric grid and the sources are placed on each grid point (voxel). Every source is defined by a current density vector with unknown moment components. The solution space includes grey matter and hippocampus. To eLORETA, the latest version of the algorithm, the brain volume consists of 6239 cortical voxels at $5 \mathrm{~mm}$ resolution. eLORETA uses a three-shell spherical head model, which provides a source localization accuracy significantly better as compared to the simple spherical shell approximation [25]. eLORETA has zero error localization in the presence of measurement and structured biological noise and introduces a weight matrix which considers the deeper sources in a more adequate way [26]. Over the years LORETA was used in several studies about Alzheimer's disease, with promising results [27-29]. Starting from cortical electrical activity estimated with LORETA, functional connectivity measurements can be performed by a toolbox recently introduced in the LORETA software. To date, this tool was employed in research about Alzheimer's disease, above all, in the case of low-density EEG. In particular, Hata et al. [30] reported a decreased lagged phase synchronization in delta band between most cortical 
regions in Alzheimer's Disease subjects in comparison to healthy controls. Moreover, Babiloni et al. [31] showed that brain functional connectivity is lower in Mild Cognitive Impairment patients compared to healthy elderly subjects.

The novelty of this paper is that brain functional connectivity is evaluated by means of eLORETA software applied to HD-EEG. The aim of this work is to study the functional connectivity through the analysis of High-Density EEG (HD-EEG) for three groups of subjects: control subjects (CNT), Mild Cognitive Impairment patients (MCI) and Alzheimer's Disease patients (AD). In particular, the purpose is to compare the results obtained using a 256-channel EEG, the corresponding 10-10 system 64-channel EEG and the corresponding 10-20 system 18-channel EEG, both of which are extracted from the 256-electrode configuration. The analysis of results suggests that the small-world properties of $\mathrm{MCI}$ and $\mathrm{AD}$ patients are weakened only when the high-density electrode configuration is used. On the contrary, the results achieved by the analysis based on the standard low-density EEG are inconsistent with the cognitive decline in $\mathrm{AD}$ patients.

\section{Materials and Methods}

The experimental setup was arranged as follows:

1. EEG data were collected from three groups of patients (CNT, MCI, and AD) by a high-density acquisition system. Starting from that, three different electrodes configurations were considered. The signals preprocessing was performed by means of MATLAB (see Section 2.1);

2. the preprocessed EEGs were used as input signals for the LORETA-KEY software. In order to quantify the functional connectivity, the Lagged Linear Connectivity (LLC) matrix was computed for each couple of regions of interest (ROIs), for a given frequency range (see Section 2.2);

3. starting from the LLC, the small-world properties of the brain networks were measured by three parameters: $\lambda, C C$, and $D$, estimated through a MATLAB toolbox (see Section 2.3).

\subsection{Data Acquisition System and Preprocessing}

EEG signals were recorded by the 256-channel HydroCel Geodesic Sensor Net, which belongs to the Geodesic EEG System (GES). Each line between electrode pairs is a geodesic, the shortest distance between two points on the surface of a sphere. The accurate geodesic tessellation of the scalp optimizes the sampling of the electrical field [32].

The high-density montage is shown in Figure 1 . The electrode impedance was kept $<50 \mathrm{k} \Omega$, according to EGI guidelines. The reference electrode was $\mathrm{Cz}$, placed in the middle of the head surface. The 18-channel and 64-channel configurations were obtained based on the 10-10 position equivalence for the HydroCel GSN [33].

The dataset consists of HD-EEG recorded at IRCCS Centro Neurolesi Bonino-Pulejo of Messina (Italy). The research was performed as stated in a protocol approved by the local Ethics Committee (Approved N. 003/17). All the subjects involved or their caregivers signed an informed consent form. EEG were acquired during eyes-closed resting conditions, for three groups of subjects: $10 \mathrm{CNT}, 21 \mathrm{MCI}$, and $9 \mathrm{AD}$. The sampling rate was $250 \mathrm{~Hz}$. The EEG were filtered at $1 \mathrm{~Hz}$ low cut-off (high-pass) and at $40 \mathrm{~Hz}$ high cut-off (low-pass). After filtering, artifactual data segments were detected by visual inspection and rejected [34]. Moreover, only 173 channels from the starting 256 were considered because the signals from electrodes placed on the face and the neck were too noisy. Two minutes of cleaned EEG were considered for each subject. EEG were finally transformed to a common average reference montage. 


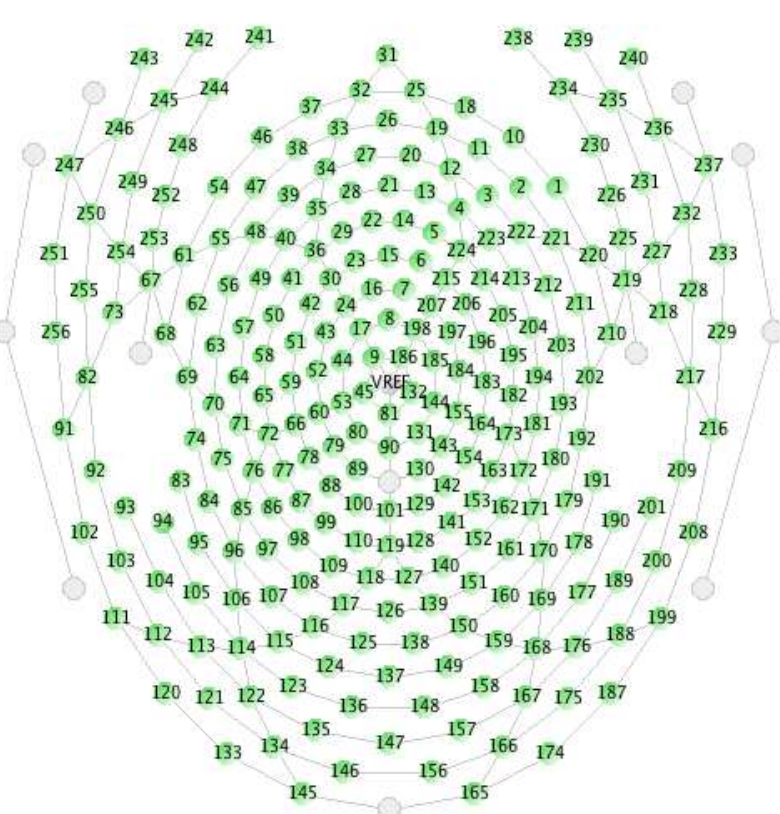

Figure 1. 256-channel HydroCel Geodesic Sensor Net (HCGSN).

\section{2. eLORETA and Lagged Linear Connectivity}

In this paper, the computation of brain connectivity was conducted by means of eLORETA algorithm implemented in the LORETA-KEY software (v20181107). LORETA is a powerful tool to analyze the activation of brain regions associated with certain functionalities. Furthermore, Pascual-Marqui et al. proposed a new method for functional connectivity measurements [35,36]. They decomposed the total connectivity into instantaneous and lagged contributions: the first one takes into account the potentials generated by distant cortical regions and conducted across head volume (the so-called "volume conduction"), while the lagged component contains almost pure physiological information.

The linear dependence between time series $X$ and $Y$ at frequency $\omega$ is defined as:

$$
F_{X, Y}(\omega)=\ln \frac{\left|\left(\begin{array}{cc}
\mathbf{S}_{Y, Y}(\omega) & \mathbf{0} \\
\mathbf{0}^{T} & \mathbf{S}_{X, X}(\omega)
\end{array}\right)\right|}{\left|\left(\begin{array}{ll}
\mathbf{S}_{Y, Y}(\omega) & \mathbf{S}_{Y, X}(\omega) \\
\mathbf{S}_{X, Y}(\omega) & \mathbf{S}_{X, X}(\omega)
\end{array}\right)\right|}
$$

where $\mathbf{S}_{X, X}, \mathbf{S}_{Y, Y}, \mathbf{S}_{X, Y}, \mathbf{S}_{Y, X}$ denote the complex valued covariance matrices and $|\mathbf{M}|$ is the determinant of $\mathbf{M}$.

Linear dependence can be expressed as the sum of the lagged linear dependence $F_{X \rightleftarrows Y}(\omega)$ and the instantaneous linear dependence $F_{X \cdot Y}(\omega)$ :

$$
F_{X, Y}(\omega)=F_{X \rightleftarrows Y}(\omega)+F_{X \cdot Y}(\omega)
$$


The instantaneous linear dependence is defined as:

$$
F_{X \cdot Y}(\omega)=\ln \frac{\left|\operatorname{Re}\left(\begin{array}{cc}
\mathbf{S}_{Y, Y}(\omega) & \mathbf{0} \\
\mathbf{0}^{T} & \mathbf{S}_{X, X}(\omega)
\end{array}\right)\right|}{\left|\operatorname{Re}\left(\begin{array}{cc}
\mathbf{S}_{Y, Y}(\omega) & \mathbf{S}_{Y, X}(\omega) \\
\mathbf{S}_{X, Y}(\omega) & \mathbf{S}_{X, X}(\omega)
\end{array}\right)\right|}
$$

where $\operatorname{Re}(\mathbf{M})$ denotes the real part of $\mathbf{M}$.

Finally, the measure of lagged linear dependence is:

$$
F_{X \rightleftarrows Y}(\omega)=F_{X, Y}(\omega)-F_{X \cdot Y}(\omega)=\ln \frac{\left\{\left|\operatorname{Re}\left(\begin{array}{ll}
\mathbf{S}_{Y, Y}(\omega) & \mathbf{S}_{Y, X}(\omega) \\
\mathbf{S}_{X, Y}(\omega) & \mathbf{S}_{X, X}(\omega)
\end{array}\right)\right| /\left|\operatorname{Re}\left(\begin{array}{cc}
\mathbf{S}_{Y, Y}(\omega) & \mathbf{0} \\
\mathbf{0}^{T} & \mathbf{S}_{X, X}(\omega)
\end{array}\right)\right|\right\}}{\left\{\left|\left(\begin{array}{ll}
\mathbf{S}_{Y, Y}(\omega) & \mathbf{S}_{Y, X}(\omega) \\
\mathbf{S}_{X, Y}(\omega) & \mathbf{S}_{X, X}(\omega)
\end{array}\right)\right| /\left|\left(\begin{array}{cc}
\mathbf{S}_{Y, Y}(\omega) & \mathbf{0} \\
\mathbf{0}^{T} & \mathbf{S}_{X, X}(\omega)
\end{array}\right)\right|\right\}}
$$

All these measures are non-negative. They are equal to zero only when there is independence (lagged, instantaneous, or both). They can also be transformed into a "squared coherence", with values in the $[0 \ldots 1]$ range.

In this paper, we used the Connectivity Toolbox implemented in LORETA-KEY software. In particular, we estimated the Lagged Linear Connectivity (LLC), which is computed by Equation (4). LLC gives a measurement of the statistical dependence among active sources, estimated by eLORETA, for each pair of cortical ROIs, at a given frequency range. Specifically, we estimated LLC for the all 84 possible ROIs defined by LORETA-KEY software. The ROIs corresponds to distinct Brodmann areas, 42 for each hemisphere. The analysis was conducted for three different electrode configurations (see Section 2.1) for the frequency range 1-40 Hz. EEG were segmented into artifact-free non-overlapping epochs of 250 samples (1s) [37]. LLC was evaluated for windows of 3 epochs, so we obtained 40 connectivity matrices for each patient. The choice of such a window length allows performance of a statistical analysis also in the event that EEG recordings are short, and the number of patients is limited.

\subsection{Complex Network Analysis}

A network is defined in graph theory as a set of nodes and the links (edges) between them, which can be directed or undirected. In our case every ROI represents a node and the graph is weighted undirected. A weighted graph provides a measure of the connection strength between nodes, so it can be used to distinguish strong connections from weak ones.

Brain functional integration is the capacity to fast combine information from distributed brain regions. The Characteristic Path Length $(\lambda)$ represents a measure of functional integration of a network [38]. $\lambda$ is defined as the average shortest path length between all pairs of nodes [13]:

$$
\lambda=\frac{1}{n} \sum_{i \in N} \frac{\sum_{j \in N, j \neq i} d_{i j}^{w}}{n-1}
$$

where $\mathrm{n}$ is the number of nodes and $d_{i j}^{w}$ is the shortest weighted path length between the nodes $i$ and $j$.

Functional segregation refers to the ability to create interconnected groups of brain regions (clusters). The Clustering Coefficient (CC) is an important parameter to quantify the presence of such groups within the network [38]. It provides a measure of the probability that the neighbors of one node become neighbors each other. The weighted Clustering Coefficient is defined as [13]:

$$
C C^{w}=\frac{1}{n} \sum_{i \in N} C C_{i}^{w}=\frac{1}{n} \sum_{i \in N} \frac{2 t_{i}^{w}}{k_{i}\left(k_{i}-1\right)}
$$


where $C C_{i}$ is the Clustering Coefficient of node $i, t_{i}$ is the number of triangles around the node $i$ and $k_{i}$ is the number of links connected to a node $i$, so that $k_{i}\left(k_{i}-1\right) / 2$ is the maximum number of possible edges can exist between neighbors of node $i$.

The Connection Density $(D)$ of a network is the fraction of actual number of edges in the graph to the total number of possible edges [17]:

$$
D=\frac{2 k}{N(N-1)}
$$

where $N$ is the number of all nodes and $k$ is the actual number of links.

A complex network characterized by high Clustering Coefficient, high Connection Density and short average path length has small-world properties.

Data processing and network analysis were conducted in a MATLAB environment (R2018a). In particular, the network parameters above-mentioned were computed by means of the Brain Connectivity Toolbox, a MATLAB toolbox for complex network analysis of structural and functional brain connectivity data sets [13].

\section{Results}

The methodology explained in Section 2 was applied to the HD-EEG database. Figure 2 displays the boxplots of the Characteristic Path Length and the Clustering Coefficient of the three groups of patients for each electrode configuration. Each boxplot is made up of 1600 parameters, 40 for each patient. Figure 2a shows that there is no clear difference of $\lambda$ between the three groups for 18 and 64 electrodes, whereas the difference becomes evident only with the HD configuration (173 electrodes).

In particular the $\lambda$ median value increases for $\mathrm{MCI}$ and even more for $\mathrm{AD}$ as compared with CNT. Figure $2 \mathrm{~b}$ highlights that the median $C C$ decreases when 64 electrodes are used, becoming more evident for 173 electrodes. For the Low-Density EEG the trend is completely different and does not correspond to the expected behavior. In order to validate the results, a statistical analysis was performed. First, a Kruskal-Wallis test [39] was carried out to determine if the samples of groups come from different distributions. Next, the post-hoc Tukey's Honestly Significant Difference (HSD) test [40] was used to perform multiple pairwise comparison of the group medians and identify which groups are statistically different. The significance level was set at $5 \%$, so that the differences between groups are statistically significant when $p<0.05$. Tables 1 and 2 show the outcome of the above-mentioned statistical analysis. In regard to $\lambda$, there is a significant difference between the all three groups only for 173 electrodes. A significant difference can be also found between CNT and AD groups when 64 sensors are considered. As for CC, it was found a statistically significant difference between CNT-MCI groups and MCI-AD groups when 18 electrodes are employed. It could seem that low-density configuration is able to detect the variations between the groups but, actually, this is not consistent with the expected trend of the parameter: in fact the median value in $\mathrm{AD}$ is higher than $\mathrm{MCI}$ and $\mathrm{CNT}$ whereas it should be lower. Finally, a statistical significant difference arises from the comparison between CNT and AD for both 64 and 173 electrodes. It is important to underline that only the results from HD-EEG are consistent with the expected behavior of the brain network, which is the weakening of small-world properties of $\mathrm{MCI}$ and $\mathrm{AD}$ patients in comparison to healthy subjects. It is noted that the variation of the network parameters was not detected correctly when we employed the standard 10-20 configuration. 

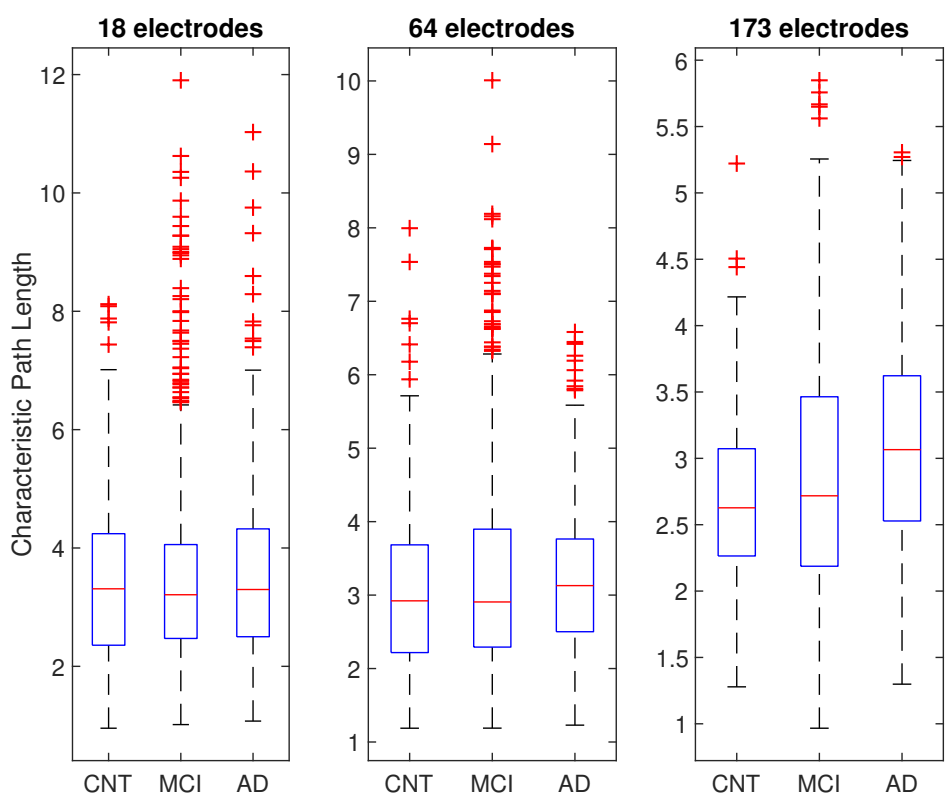

(a)
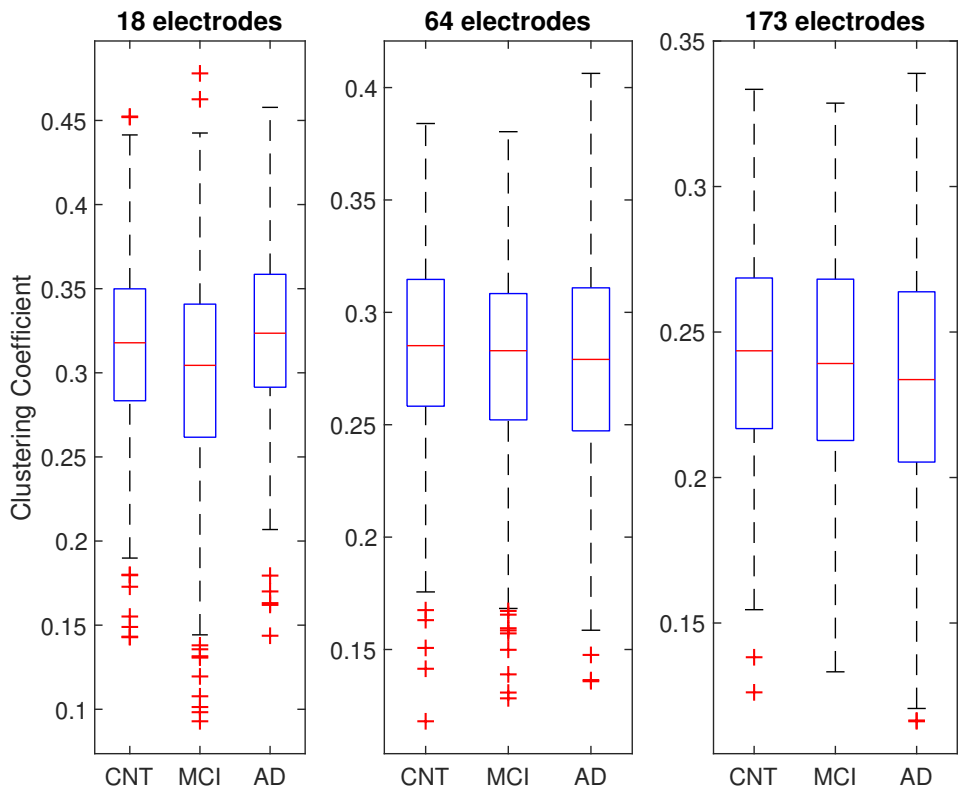

(b)

Figure 2. (a) Boxplot of the Characteristic Path Length of CNT, MCI, and AD; (b) boxplot of the Clustering Coefficient of CNT, MCI, and AD. Both are computed for three electrode configurations. The bottom and the top edges of the boxes indicate the first and the third quartile, respectively; the segment inside the boxes represents the median and the "whiskers" below and above the boxes are the minimum and maximum values of the distribution. The stars outside the whiskers are considered outliers. 
Table 1. Results of Kruskal-Wallis test.

\begin{tabular}{ccccccccc}
\hline \multicolumn{2}{c}{$\begin{array}{c}\text { Characteristic Path Length } \\
p \text {-Value }\end{array}$} & \multicolumn{2}{c}{$\begin{array}{c}\text { Clustering Coefficient } \\
p \text {-Value }\end{array}$} & \multicolumn{3}{c}{$\begin{array}{c}\text { Connection Density } \\
p \text {-Value }\end{array}$} \\
\hline $\mathbf{1 8}$ & $\mathbf{6 4}$ & $\mathbf{1 7 3}$ & $\mathbf{1 8}$ & $\mathbf{6 4}$ & $\mathbf{1 7 3}$ & $\mathbf{1 8}$ & $\mathbf{6 4}$ & $\mathbf{1 7 3}$ \\
\hline 0.4345 & 0.0130 & $1.38 \times 10^{-12}$ & $6.24 \times 10^{-10}$ & 0.0282 & 0.0481 & 0.8780 & 0.9870 & 0.9965 \\
\hline
\end{tabular}

Table 2. Results of Tukey's HSD post-hoc test.

\begin{tabular}{lcccccc}
\hline \multirow{2}{*}{ Subjects } & \multicolumn{3}{c}{ Characteristic Path Length } & \multicolumn{3}{c}{$\begin{array}{c}\text { Clustering Coefficient } \\
\boldsymbol{p} \text {-Value }\end{array}$} \\
\cline { 2 - 7 } & $\mathbf{1 8}$ & $\mathbf{6 4}$ & $\mathbf{1 7 3}$ & $\mathbf{1 8}$ & $\mathbf{6 4}$ & $\mathbf{1 7 3}$ \\
\hline CNT-MCI & 0.9974 & 0.1835 & 0.0101 & $2.8 \times 10^{-4}$ & 0.0613 & 0.3000 \\
MCI-AD & 0.4507 & 0.2054 & $8.6 \times 10^{-8}$ & $3.0 \times 10^{-9}$ & 0.7935 & 0.3353 \\
CNT-AD & 0.5132 & 0.0091 & $9.5 \times 10^{-10}$ & 0.0918 & 0.0377 & 0.0368 \\
\hline
\end{tabular}

The Connection Density was computed as a function of the threshold applied to the weighted connection matrix. Obviously, the network will be totally connected if the threshold is set to 0 and disconnected if the threshold is set to 1 . Figure 3 shows the mean value of D of each group of subjects for the three sensors configurations. AD and MCI patients should have lower network density than healthy controls. This trend is visible only when we used at least 64 sensors and becomes more evident with 173 electrodes. The statistical analysis shows that the data of the three groups come from the same distribution, so there is not a significant statistical difference between them (Table 1). Nevertheless, if we consider some patients individually, there is a reduction of the connections between the brain regions when 173 electrodes were employed. As an example, Figures 4-6 display the links between the network nodes, representing the 84 ROIs, of one subject for each group, for the three electrode configurations. The images were obtained by thresholding the weighted connection matrix at 0.3 : at this value the corresponding network is moderately connected (Figure 3). It is evident that there is a clear reduction in the network links for MCI and AD just when 173 sensors are used. Specifically, there are no connections in the posterior regions for MCI and AD. This outcome agrees with recent studies reporting a reduction in functional connectivity within posterior areas in Alzheimer's Disease [41,42]. For 18 and 64 electrodes the pictures of the three groups are indistinguishable. It can be supposed that 64 sensors are not sufficient for a correct estimation of functional connectivity. This topic needs further investigation in the future. 


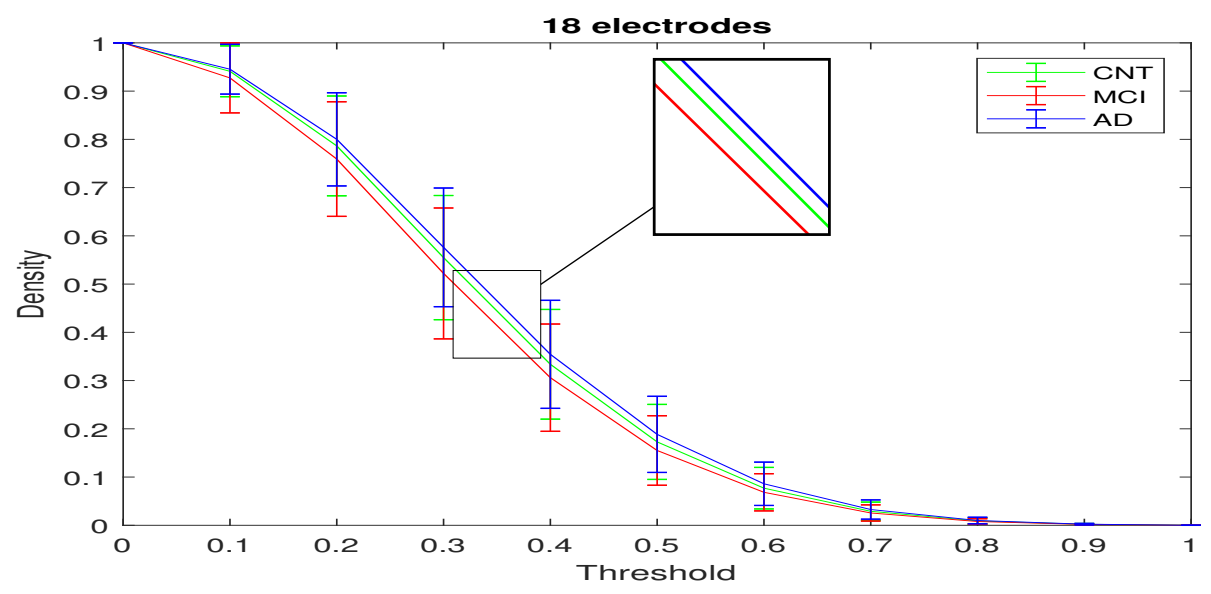

(a)

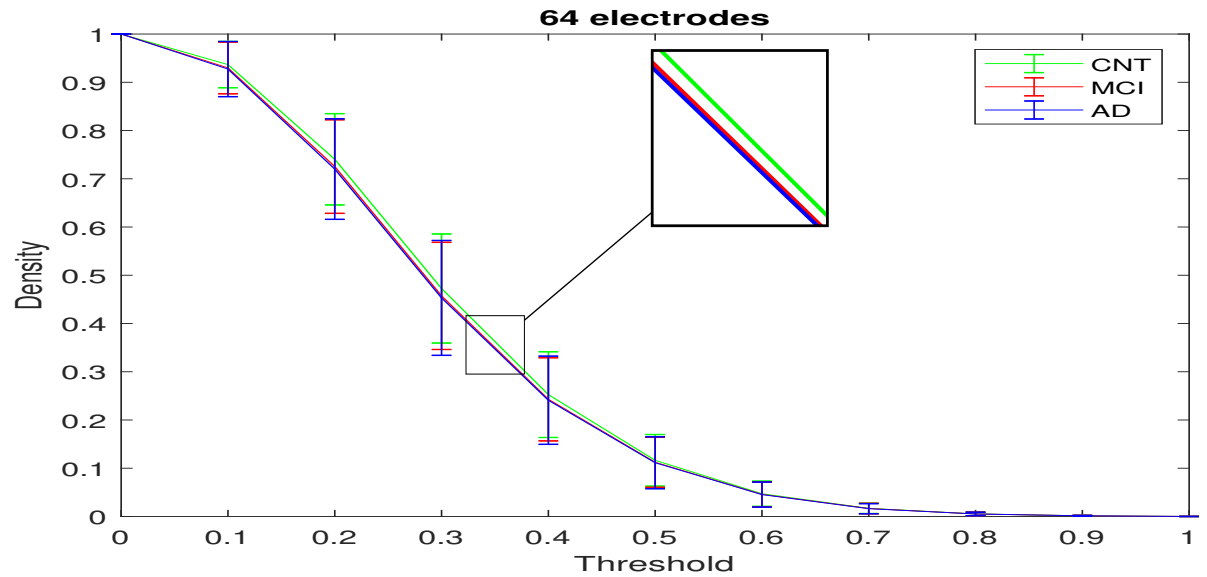

(b)

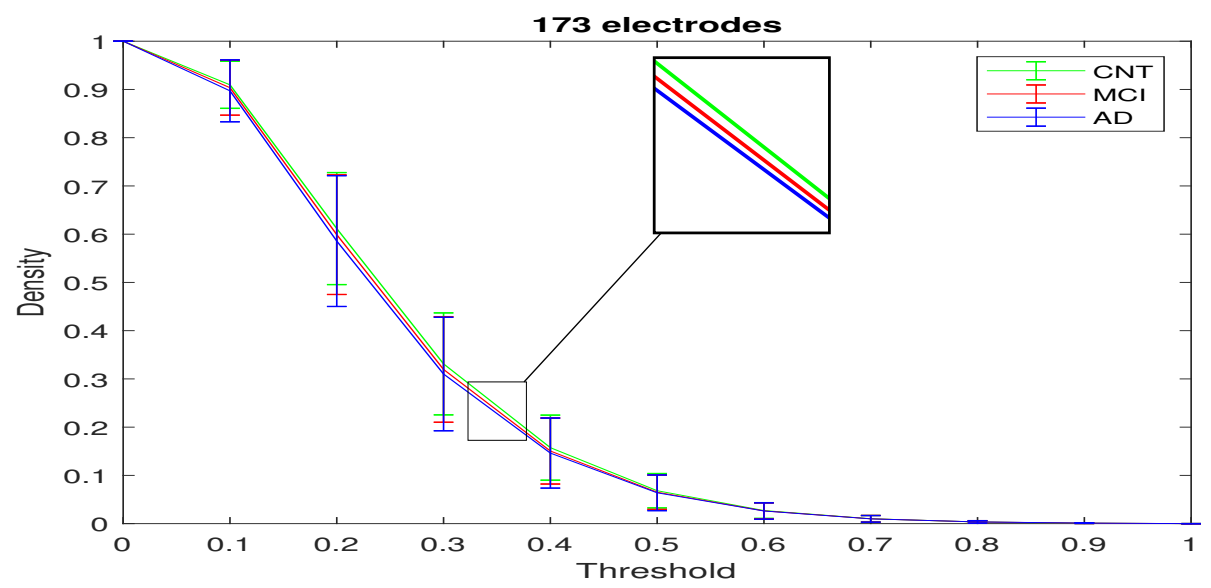

(c)

Figure 3. Mean values of the Connection Density (D) computed by thresholding the connectivity matrix of CNT, MCI, and AD for: (a) 18 electrodes, (b) 64 electrodes and (c) 173 electrodes. The vertical segments represent the standard deviation of $\mathrm{D}$. 


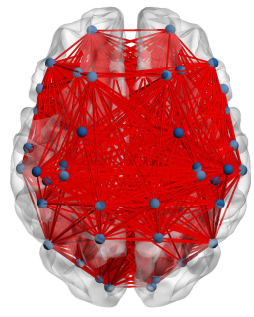

(a)

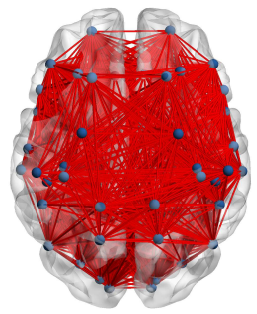

(b)

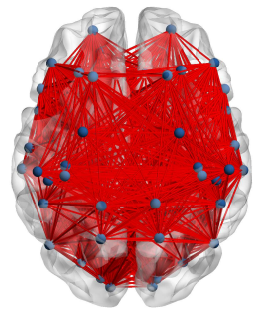

(c)

Figure 4. Connections between ROIs for 18 electrodes of: (a) CNT, (b) MCI and (c) AD.

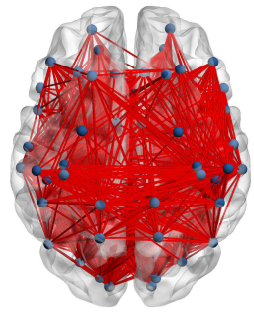

(a)

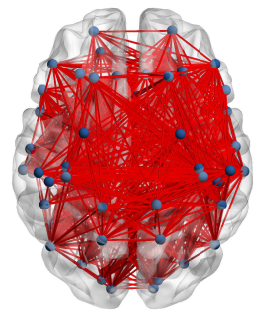

(b)

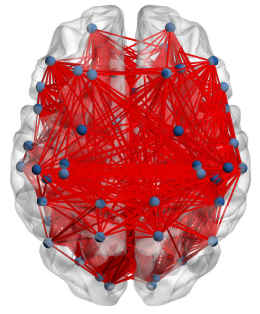

(c)

Figure 5. Connections between ROIs for 64 electrodes of: (a) CNT, (b) MCI and (c) AD.

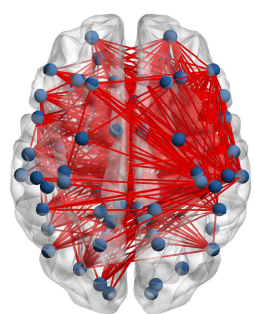

(a)

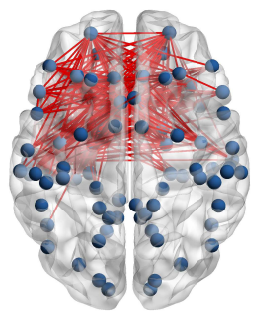

(b)

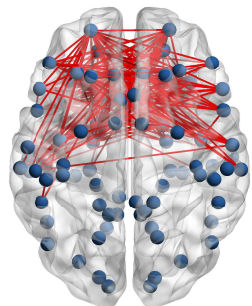

(c)

Figure 6. Connections between ROIs for 173 electrodes of: (a) CNT, (b) MCI and (c) AD.

\section{Discussion and Conclusions}

Alzheimer's disease is a neurological disorder characterized by a progressive impairment of cognitive functions. In its early stages, it does not interfere with daily life significantly. As the disease advances, symptoms become more severe and, eventually, patients cannot take care of themselves any longer. From a neurological point of view, brain connections are weakened or completely lost because of the death of neurons. EEG is a powerful tool to explore brain functioning and detect any alterations in the presence of pathological conditions [43-46]. Recently, graph theory has widely been employed to quantify the brain functional connectivity; it has also been proved that brain networks are characterized by small-world architecture. Several techniques have been employed for evaluating the functional connectivity during the last two decades. Bastos and Schoffelen [47] provided a review of some methods, such as coherence, phase synchronization, phase-slope index. Rosales et al. [48] proposed a new implementation of the Synchronization Likelihood algorithm, which improves significantly its computational and memory performance. Lombardi et al. [49] described a new synchronization-based metric which is more sensitive to nonlinear coupling phenomena between time series and more robust with respect to noise. In this work we study the brain network behavior for 10 healthy subjects (CNT), 
$21 \mathrm{MCI}$, and $9 \mathrm{AD}$ patients. The brain functional connectivity was quantified by the computation of the Lagged Linear Connectivity parameter, which can be measured through the toolbox implemented in the eLORETA software. This index provides a measure of the true physiological connections, excluding instantaneous zero-lag contribution related to the volume conduction [36]. The novelty is the comparison between HD-EEG and LD-EEG results evaluated with this method: in particular, the network parameters were estimated for 84 ROIs considering 18, 64, and 173 electrodes, extracted by the starting 256-channel EEG. From the analysis of the three network parameters we focused on, arise that: the Characteristic Path Length of MCI and AD patients is higher than CNT for the 173 electrode configuration, whereas it does not show a clear difference between the three groups of patients for 18 electrodes; the Clustering Coefficient of MCI and AD decreases when 173 sensors are employed but it increases for AD with 18 electrodes; as reported in Table 3, which refers to Figure 3, the Connection Density decreases for $\mathrm{MCI}$ and $\mathrm{AD}$ with 173 electrodes; on the contrary it increases for AD when 18 sensors are used. The statistical analysis validates the results for $\lambda$ and $C C$, according to which the small-world properties of MCI and AD patients are weakened just when the high-density electrode configuration is used. When the study is based on the standard low-density EEG, the results are not consistent with the behavior of the brain network affected by this pathology. Therefore, the current study suggests that it is advisable to use HD-EEG to achieve a better accuracy of results. These findings are also supported by the studies reported in [9-12], according to which the localization accuracy of the epileptogenic zones, reconstructed by LORETA, improves when a high-density montage is used. As a consequence, the more the active-source localization is precise, the more the computation of the functional connectivity between brain regions is reliable. Although our results appear interesting, some limitations should be addressed. In particular, EEG recordings of a greater duration could be detected: in this case, it could be investigated if the EEG epochs and windows lengths affected the network parameters [50]. The methodology needs to be tested on a larger number of subjects, to confirm our results. Moreover, an intra-subject longitudinal study could be carried out to analyze the evolution of the disease in each single patient over time. The complex network analysis could also be performed together with the reconstruction of brain active sources for each EEG frequency sub-band (delta, theta, alpha, beta, and gamma). The integration of these analysis could provide a more complete vision of the brain behavior, resulting in a valid instrument for quantifying the loss of brain connections typical of Alzheimer's Disease. As a further development, future research could be performed, for example, with more advanced methodological improvements, combining the transcranial magnetic stimulation (TMS) with HD-EEG [51]. In conclusion, our study shows that HD-EEG seems to reveal resting-state connectivity alterations in AD patients, so this method could be a useful tool to predict the disease severity and improve diagnosis, clinical and therapeutic management.

Table 3. Mean Connection Density values of CNT, MCI, and AD for all electrode configurations for three threshold values.

\begin{tabular}{|c|c|c|c|c|c|c|c|c|c|}
\hline \multirow{3}{*}{ THRESHOLD } & \multicolumn{9}{|c|}{ DENSITY } \\
\hline & \multicolumn{3}{|c|}{18} & \multicolumn{3}{|c|}{64} & \multicolumn{3}{|c|}{173} \\
\hline & CNT & MCI & $\mathrm{AD}$ & CNT & MCI & $\mathrm{AD}$ & CNT & MCI & AD \\
\hline 0.3 & 0.5549 & 0.5221 & 0.5761 & 0.4725 & 0.4572 & 0.4530 & 0.3312 & 0.3196 & 0.3101 \\
\hline 0.5 & 0.1729 & 0.1551 & 0.1885 & 0.1164 & 0.1123 & 0.1114 & 0.0682 & 0.0651 & 0.0639 \\
\hline 0.7 & 0.0290 & 0.0256 & 0.0327 & 0.0168 & 0.0165 & 0.0161 & 0.0101 & 0.0100 & 0.0099 \\
\hline
\end{tabular}

Author Contributions: Conceptualization, F.L.F.; Methodology, F.L.F.; Software, S.D.; Validation, S.D.; Formal analysis, F.L.F.; Investigation, S.D. and F.C.M.; Resources, S.M.; Data curation, S.M.

Funding: This research received no external funding. 
Acknowledgments: The authors thank the doctors of IRCCS Centro Neurolesi Bonino-Pulejo of Messina (Italy) for their insightful comments and suggestions. The authors also thank Nadia Mammone for her support to the organization of the database.

Conflicts of Interest: The authors declare no conflict of interest.

\section{References}

1. Labate, D.; La Foresta, F.; Palamara, I.; Morabito, G.; Bramanti, A.; Zhang, Z.; Morabito, F.C. EEG complexity modifications and altered compressibility in mild cognitive impairment and Alzheimer's disease. In Recent Advances of Neural Network Models and Applications; Springer: Berlin/Heidelberg, Germany, 2014; pp. 163-173.

2. Morabito, F.C.; Campolo, M.; Ieracitano, C.; Ebadi, J.M.; Bonanno, L.; Bramanti, A.; Desalvo, S.; Mammone, N.; Bramanti, P. Deep convolutional neural networks for classification of mild cognitive impaired and Alzheimer's disease patients from scalp EEG recordings. In Proceedings of the 2016 IEEE 2nd International Forum on Research and Technologies for Society and Industry Leveraging a Better Tomorrow (RTSI), Bologna, Italy, 7-9 September 2016; pp. 1-6.

3. Mammone, N.; De Salvo, S.; Bonanno, L.; Ieracitano, C.; Marino, S.; Marra, A.; Bramanti, A.; Morabito, F.C. Brain Network Analysis of Compressive Sensed High-Density EEG Signals in AD and MCI Subjects. IEEE Trans. Ind. Inform. 2019, 15, 527-536. [CrossRef]

4. Wang, G.; Ren, D. Effect of brain-to-skull conductivity ratio on EEG source localization accuracy. BioMed Res. Int. 2013, 2013. [CrossRef] [PubMed]

5. Spitzer, A.R.; Cohen, L.G.; Fabrikant, J.; Hallett, M. A method for determining optimal interelectrode spacing for cerebral topographic mapping. Electroencephalogr. Clin. Neurophysiol. 1989, 72, 355-361. [CrossRef]

6. Tucker, D.M. Spatial sampling of head electrical fields: The geodesic sensor net. Electroencephalogr. Clin. Neurophysiol. 1993, 87, 154-163. [CrossRef]

7. Freeman, W.J.; Holmes, M.D.; Burke, B.C.; Vanhatalo, S. Spatial spectra of scalp EEG and EMG from awake humans. Clin. Neurophysiol. 2003, 114, 1053-1068. [CrossRef]

8. Srinivasan, R.; Tucker, D.M.; Murias, M. Estimating the spatial Nyquist of the human EEG. Behav. Res. Methods Instrum. Comput. 1998, 30, 8-19. [CrossRef]

9. Wang, G.; Worrell, G.; Yang, L.; Wilke, C.; He, B. Interictal spike analysis of high-density EEG in patients with partial epilepsy. Clin. Neurophysiol. 2011, 122, 1098-1105. [CrossRef] [PubMed]

10. Sohrabpour, A.; Lu, Y.; Kankirawatana, P.; Blount, J.; Kim, H.; He, B. Effect of EEG electrode number on epileptic source localization in pediatric patients. Clin. Neurophysiol. 2015, 126, 472-480. [CrossRef]

11. Song, J.; Davey, C.; Poulsen, C.; Luu, P.; Turovets, S.; Anderson, E.; Li, K.; Tucker, D. EEG source localization: Sensor density and head surface coverage. J. Neurosci. Methods 2015, 256, 9-21. [CrossRef]

12. Staljanssens, W.; Strobbe, G.; Van Holen, R.; Birot, G.; Gschwind, M.; Seeck, M.; Vandenberghe, S.; Vulliémoz, S.; van Mierlo, P. Seizure onset zone localization from ictal high-density EEG in refractory focal epilepsy. Brain Topogr. 2017, 30, 257-271. [CrossRef]

13. Rubinov, M.; Sporns, O. Complex network measures of brain connectivity: Uses and interpretations. Neuroimage 2010, 52, 1059-1069. [CrossRef] [PubMed]

14. Coito, A.; Genetti, M.; Pittau, F.; Iannotti, G.R.; Thomschewski, A.; Höller, Y.; Trinka, E.; Wiest, R.; Seeck, M.; Michel, C.M.; et al. Altered directed functional connectivity in temporal lobe epilepsy in the absence of interictal spikes: A high density EEG study. Epilepsia 2016, 57, 402-411. [CrossRef] [PubMed]

15. Astolfi, L.; De Vico Fallani, F.; Cincotti, F.; Mattia, D.; Marciani, M.; Bufalari, S.; Salinari, S.; Colosimo, A.; Ding, L.; Edgar, J.; et al. Imaging functional brain connectivity patterns from high-resolution EEG and fMRI via graph theory. Psychophysiology 2007, 44, 880-893. [CrossRef] [PubMed]

16. Bassett, D.S.; Bullmore, E. Small-world brain networks. Neuroscientist 2006, 12, 512-523. [CrossRef] [PubMed]

17. Bullmore, E.; Sporns, O. Complex brain networks: Graph theoretical analysis of structural and functional systems. Nat. Rev. Neurosci. 2009, 10, 186. [CrossRef]

18. Jeong, J. EEG dynamics in patients with Alzheimer's disease. Clin. Neurophysiol. 2004, 115, 1490-1505. [CrossRef]

19. König, T.; Prichep, L.; Dierks, T.; Hubl, D.; Wahlund, L.; John, E.; Jelic, V. Decreased EEG synchronization in Alzheimer's disease and mild cognitive impairment. Neurobiol. Aging 2005, 26, 165-171. [CrossRef] 
20. Sankari, Z.; Adeli, H.; Adeli, A. Intrahemispheric, interhemispheric, and distal EEG coherence in Alzheimer's disease. Clin. Neurophysiol. 2011, 122, 897-906. [CrossRef]

21. Stam, C.J.; Jones, B.; Nolte, G.; Breakspear, M.; Scheltens, P. Small-world networks and functional connectivity in Alzheimer's disease. Cereb. Cortex 2006, 17, 92-99. [CrossRef]

22. He, Y.; Chen, Z.; Gong, G.; Evans, A. Neuronal networks in Alzheimer's disease. Neuroscientist 2009, 15, 333-350.

23. Wang, R.; Wang, J.; Yu, H.; Wei, X.; Yang, C.; Deng, B. Decreased coherence and functional connectivity of electroencephalograph in Alzheimer's disease. Chaos Interdiscip. J. Nonlinear Sci. 2014, 24, 033136. [CrossRef] [PubMed]

24. Mammone, N.; Ieracitano, C.; Adeli, H.; Bramanti, A.; Morabito, F.C. Permutation Jaccard distance-based hierarchical clustering to estimate EEG network density modifications in MCI subjects. IEEE Trans. Neural Networks Learn. Syst. 2018, 29, 5122-5135. [CrossRef] [PubMed]

25. Fuchs, M.; Kastner, J.; Wagner, M.; Hawes, S.; Ebersole, J.S. A standardized boundary element method volume conductor model. Clin. Neurophysiol. 2002, 113, 702-712. [CrossRef]

26. Pascual-Marqui, R.D. Discrete, 3 D distributed, linear imaging methods of electric neuronal activity. Part 1 : Exact, zero error localization. arXiv 2007, arXiv:0710.3341.

27. Rossini, P.; Del Percio, C.; Pasqualetti, P.; Cassetta, E.; Binetti, G.; Dal Forno, G.; Ferreri, F.; Frisoni, G.; Chiovenda, P.; Miniussi, C.; et al. Conversion from mild cognitive impairment to Alzheimer's disease is predicted by sources and coherence of brain electroencephalography rhythms. Neuroscience 2006, 143, 793-803. [CrossRef] [PubMed]

28. Gianotti, L.R.; Künig, G.; Lehmann, D.; Faber, P.L.; Pascual-Marqui, R.D.; Kochi, K.; Schreiter-Gasser, U. Correlation between disease severity and brain electric LORETA tomography in Alzheimer's disease. Clin. Neurophysiol. 2007, 118, 186-196. [CrossRef] [PubMed]

29. Babiloni, C.; Del Percio, C.; Lizio, R.; Noce, G.; Cordone, S.; Lopez, S.; Soricelli, A.; Ferri, R.; Pascarelli, M.T.; Nobili, F.; et al. Abnormalities of cortical neural synchronization mechanisms in subjects with mild cognitive impairment due to Alzheimer's and Parkinson's Diseases: An EEG study. J. Alzheimer's Dis. 2017, 59, 339-358. [CrossRef] [PubMed]

30. Hata, M.; Kazui, H.; Tanaka, T.; Ishii, R.; Canuet, L.; Pascual-Marqui, R.D.; Aoki, Y.; Ikeda, S.; Kanemoto, H.; Yoshiyama, K.; et al. Functional connectivity assessed by resting state EEG correlates with cognitive decline of Alzheimer's disease-An eLORETA study. Clin. Neurophysiol. 2016, 127, 1269-1278. [CrossRef]

31. Babiloni, C.; Del Percio, C.; Lizio, R.; Noce, G.; Lopez, S.; Soricelli, A.; Ferri, R.; Pascarelli, M.T.; Catania, V.; Nobili, F.; et al. Functional cortical source connectivity of resting state electroencephalographic alpha rhythms shows similar abnormalities in patients with mild cognitive impairment due to Alzheimer's and Parkinson's diseases. Clin. Neurophysiol. 2018, 129, 766-782. [CrossRef]

32. Geodesics, E. Geodesic Sensor Net Technical Manual; Electrical Geodesics: Eugene, OR, USA, 2007.

33. Luu, P.; Ferree, T. Determination of the HydroCel Geodesic Sensor Nets' Average Electrode Positions and Their 10-10 International Equivalents; Technical Note; Electrical Geodesics, Inc.: Eugene, OR, USA, 2005.

34. Mammone, N.; Morabito, F.C. Independent component analysis and high-order statistics for automatic artifact rejection. In Proceedings of the 2005 IEEE International Joint Conference on Neural Networks, Montreal, QC, Canada, 31 July-4 August 2005; Volume 4, pp. 2447-2452.

35. Pascual-Marqui, R.D. Instantaneous and lagged measurements of linear and nonlinear dependence between groups of multivariate time series: Frequency decomposition. arXiv 2007, arXiv:0711.1455.

36. Pascual-Marqui, R.D.; Lehmann, D.; Koukkou, M.; Kochi, K.; Anderer, P.; Saletu, B.; Tanaka, H.; Hirata, K.; John, E.R.; Prichep, L.; et al. Assessing interactions in the brain with exact low-resolution electromagnetic tomography. Philos. Trans. R. Soc. A Math. Phys. Eng. Sci. 2011, 369, 3768-3784. [CrossRef] [PubMed]

37. Nunez, P.L.; Srinivasan, R.; Westdorp, A.F.; Wijesinghe, R.S.; Tucker, D.M.; Silberstein, R.B.; Cadusch, P.J. EEG coherency: I: Statistics, reference electrode, volume conduction, Laplacians, cortical imaging, and interpretation at multiple scales. Electroencephalogr. Clin. Neurophysiol. 1997, 103, 499-515. [CrossRef]

38. Watts, D.J.; Strogatz, S.H. Collective dynamics of 'small-world'networks. Nature 1998, 393, 440. [CrossRef] [PubMed]

39. Breslow, N. A generalized Kruskal-Wallis test for comparing K samples subject to unequal patterns of censorship. Biometrika 1970, 57, 579-594. [CrossRef] 
40. Hochberg, J.; TAMHANE, A.C. Multiple Comparison Procedures; Technical Report; John Wiley \& Sons: Hoboken, NJ, USA, 1987.

41. McCarthy, P.; Benuskova, L.; Franz, E.A. The age-related posterior-anterior shift as revealed by voxelwise analysis of functional brain networks. Front. Aging Neurosci. 2014, 6, 301. [CrossRef] [PubMed]

42. Yu, M.; Gouw, A.A.; Hillebrand, A.; Tijms, B.M.; Stam, C.J.; van Straaten, E.C.; Pijnenburg, Y.A. Different functional connectivity and network topology in behavioral variant of frontotemporal dementia and Alzheimer's disease: An EEG study. Neurobiol. Aging 2016, 42, 150-162. [CrossRef] [PubMed]

43. Mammone, N.; Morabito, F.C.; Principe, J.C. Visualization of the short term maximum lyapunov exponent topography in the epileptic brain. In Proceedings of the 2006 International Conference of the IEEE Engineering in Medicine and Biology SocietyE, New York, NY, USA, 30 August-3 September 2006; pp. 4257-4260.

44. Mammone, N.; Principe, J.C.; Morabito, F.C.; Shiau, D.S.; Sackellares, J.C. Visualization and modelling of STLmax topographic brain activity maps. J. Neurosci. Methods 2010, 189, 281-294. [CrossRef] [PubMed]

45. Mammone, N.; Inuso, G.; La Foresta, F.; Versaci, M.; Morabito, F.C. Clustering of entropy topography in epileptic electroencephalography. Neural Comput. Appl. 2011, 20, 825-833. [CrossRef]

46. Mammone, N.; De Salvo, S.; Ieracitano, C.; Marino, S.; Cartella, E.; Bramanti, A.; Giorgianni, R.; Morabito, F. Compressibility of High-Density EEG Signals in Stroke Patients. Sensors 2018, 18, 4107. [CrossRef]

47. Bastos, A.M.; Schoffelen, J.M. A tutorial review of functional connectivity analysis methods and their interpretational pitfalls. Front. Syst. Neurosci. 2016, 9, 175. [CrossRef]

48. Rosales, F.; Garcia-Dopico, A.; Bajo, R.; Nevado, A. An efficient implementation of the synchronization likelihood algorithm for functional connectivity. Neuroinformatics 2015, 13, 245-258. [CrossRef] [PubMed]

49. Lombardi, A.; Tangaro, S.; Bellotti, R.; Bertolino, A.; Blasi, G.; Pergola, G.; Taurisano, P.; Guaragnella, C. A novel synchronization-based approach for functional connectivity analysis. Complexity 2017, 2017. [CrossRef]

50. Tzimourta, K.D.; Giannakeas, N.; Tzallas, A.T.; Astrakas, L.G.; Afrantou, T.; Ioannidis, P.; Grigoriadis, N.; Angelidis, P.; Tsalikakis, D.G.; Tsipouras, M.G. EEG Window Length Evaluation for the Detection of Alzheimer's Disease over Different Brain Regions. Brain Sci. 2019, 9, 81. [CrossRef] [PubMed]

51. Miniussi, C.; Thut, G. Combining TMS and EEG offers new prospects in cognitive neuroscience. Brain Topogr. 2010, 22, 249. [CrossRef] [PubMed]

(C) 2019 by the authors. Licensee MDPI, Basel, Switzerland. This article is an open access article distributed under the terms and conditions of the Creative Commons Attribution (CC BY) license (http://creativecommons.org/licenses/by/4.0/). 